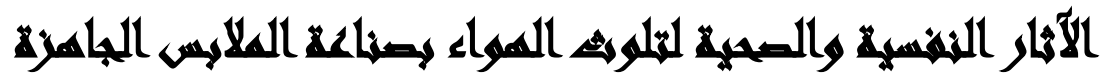

\section{$\left[\begin{array}{ll}1 & Y\end{array}\right]$}

أحمد مصطفى العتيق(')- أحمد عصمت شومان(Y) - سارة مصطفى محمد

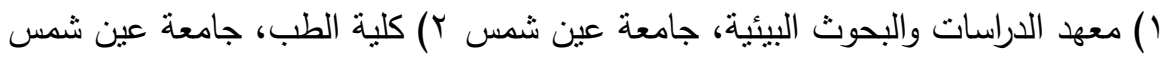

\section{المستخلس}

إزداد إهتمام الدول وخاصة المتقدمة منها بعد الثورة الصناعية، بالملوثات البيئية التي تؤثر

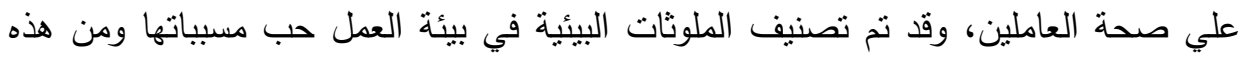

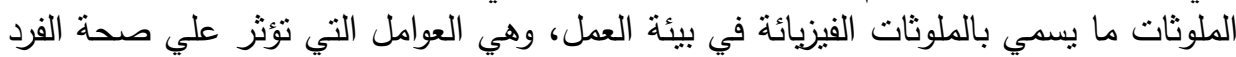

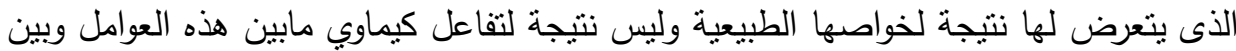

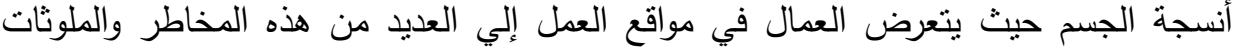

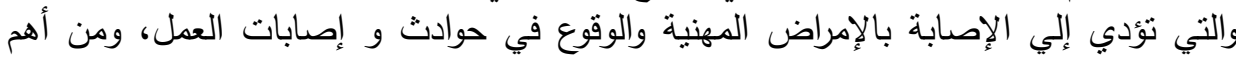

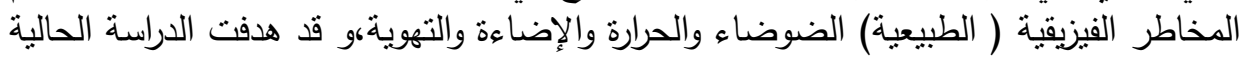
إلي التعرف علي بعض المشكلات الصحية والنفسية المرتبطة بتلوث الهواء باءة بمصانع الغية الغزل

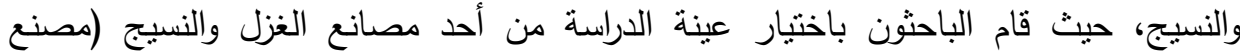

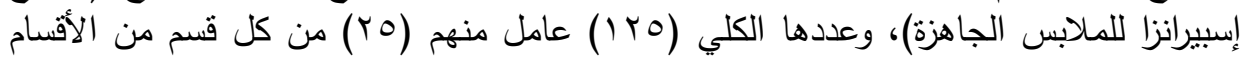

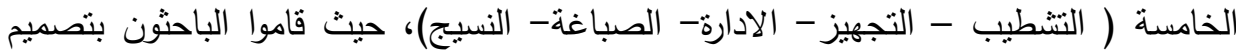

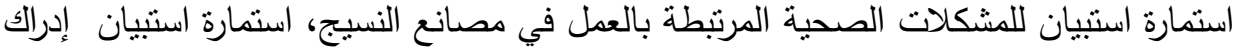
المخاطر البيئية، مقياس الضغوط المهنية، مقياس إيزنك للشخصية، فيلة في جمع البيانات وتطبيقها

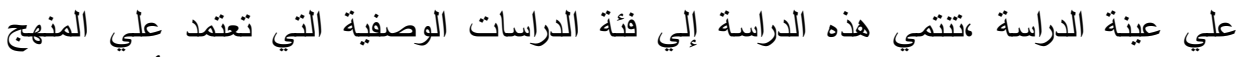

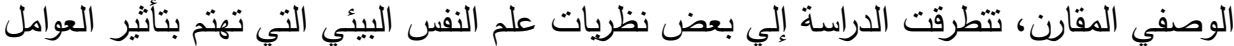
النفسية والفيزيقية في بيئة العمل مثل نظرية المشقة البيئية، منحني الاستثارة، المشقة البيائية،

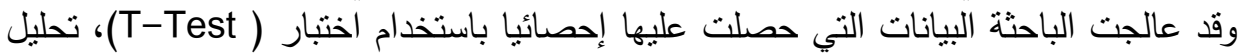

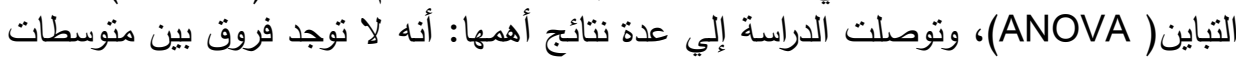

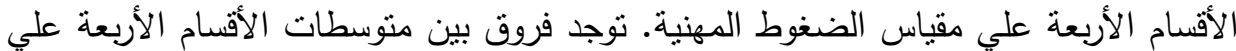
استبيان المخاطر البيئية. توجد فروق بين متوسطات الأقسام الأربعة علي الأستبيان الصدي الصدي

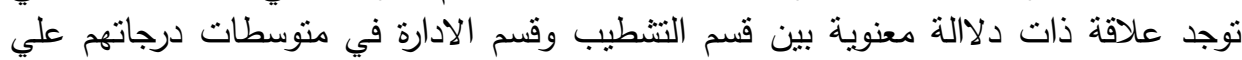
مقياس إيزنك للثخصية. 
تفعيل دور الأمن الصناعى والسلامة والصحة المهنية فى حماية العمال من المخاطر العمل

ضرورة التمام المسئولين وأصحاب المؤسسات الصناعية بتزويد العمال بأدوات الوقاية من التلوث ( الكمامات - سداد الأذن - النظارات المهنية - القفازات وبدل العنات العمل و غيرها )

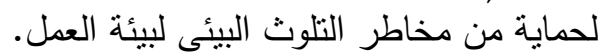

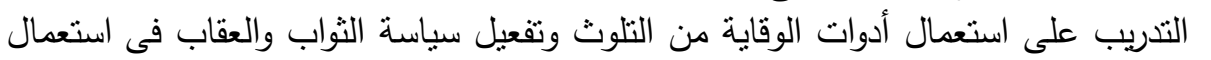

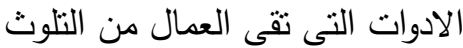

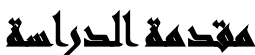

تعتبر صناعة الغزل والنسيج والملابس الجاهزة صناعة إستراتيجية كبري لاتستغني عنها أي دولة نظراً لاهمتيتها للإنسان، حيث أن القدرة في الحصول علي احتباجات الإنسان من هذه

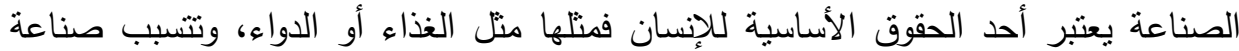

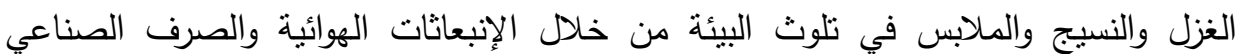

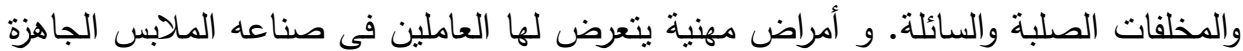

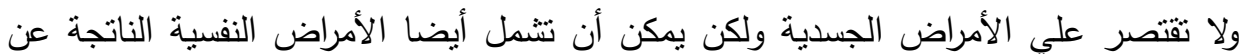

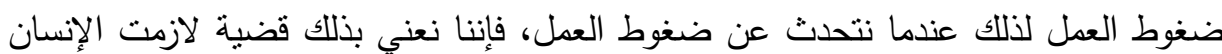
منذ وجوده على الأرض، فقد وجد ليعمل وكان هذا العمل ولا يزال مصدر الثقاء وذالك مصداقاً

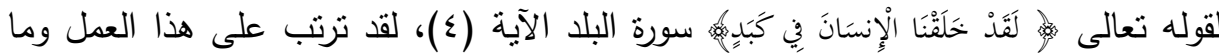

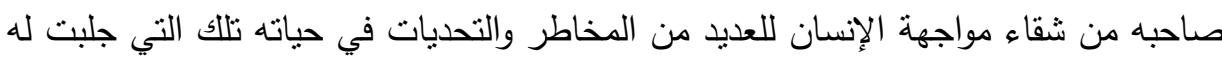

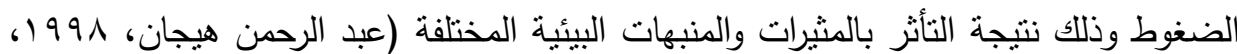
ص rا 1) (محمود وهذه الضغوط جعلت الفرد يعيش حالة قلق وتوتر وانفعال مما أثر على مهام

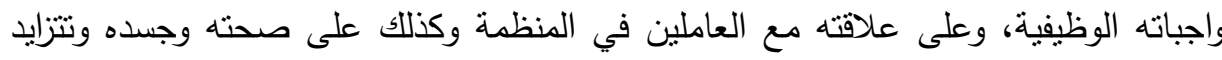
الضغوط التي تقرضها بيئة العمل مع تزايد التقدم التكنولوجي والهنافسة العالمية (محمود

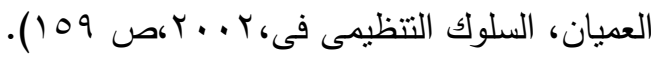




\section{هingen}

علي الرغم من أهمية صناعة النسيج والملابس الجاهزة وآثارها الإيجابية عليي نتوع مصادر العمل والاخل وقدرتها علي إستيعاب الزيادة الكبيرة من الإيدي العاملة إلا أنه ينجم عنه الهـ

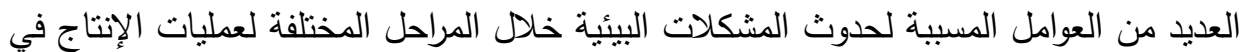
أقسامها المختلفة" الغزل والنسيج والصباغة والجهيز" والتي يكون لها إنعكاسات خطيرة علي لهي

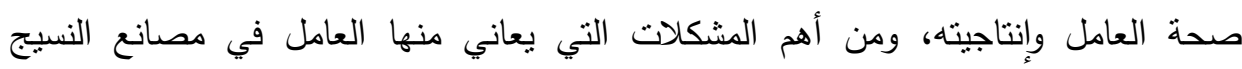
والملابس الجاهزة في المدن الصناعية المصرية أستحالة عمل معالجات مركزية لمخلفات الصباغة والتباعد الجغرافي للثركات العاملة في مجال صباغة النسيج وصناعة الملابس وكذلك عدم إلتزام العديد من الثركات بتطبيق القواعد المنصوص عليها بالقوانين واللوائح البيئية

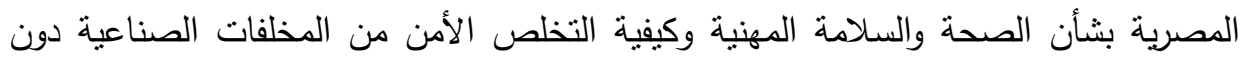

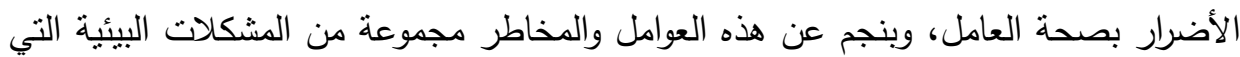
تواجه العاملين داخل المصانع ومن أهمها مشكلات تلوث الهواء( مشكلات الغبار) التي تسبب لهواء

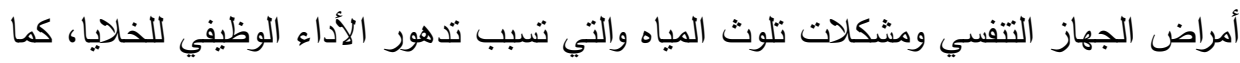
ينتج عن المخلفات الصلبة آثار سلبية علي صحة العمال لأنها تحتوي علي مواد سامة. وفى سبيل ذلك يستعرض الباحثون بعض البحوث والدراسات التى اجريت على صناعة النسيج وبعض الصناعات المماثلة:

1 - محمد محمد سالم(ب . Y): تأثثير المشروعات الصناعية علي تلوث البيئة، هدفت الدراسة

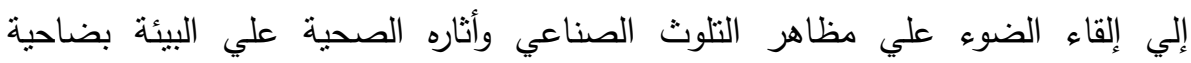
حلوان،التعرف علي أنواع المخلفات التي تفرزها المشروعات الصناعية بالمنطقة، تتفق هذه الهن

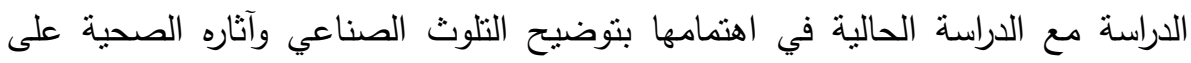
البيئة.

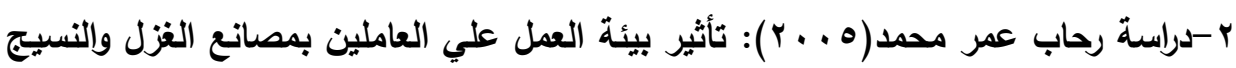

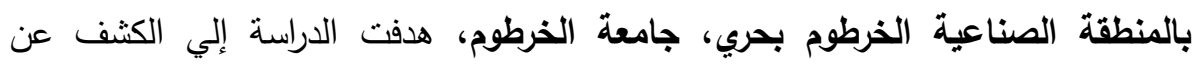


العوامل الموجودة في بيئة العمل وتأثثراتها علي صحة العامل، ومعرفة مدي نطبيق وسائل الحماية للحد من تأثثرات تلك العوامل الضارة. تتفق هذه الدراسة مع الدراسة الحالة في الهدف حيث تهدف الدراستان إلى توضيح تأثير

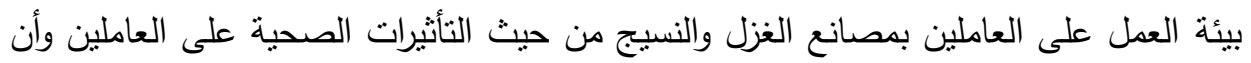
تطبيق وسائل الحماية تعد من العوامل التى تقلل من التأثيرات العوامل الضارة.

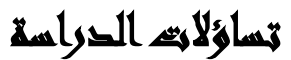

• ما المشكلات النفسية التي تواجه العاملين في صناعة الملابس الجاهزة ؟

• ما المشكلات الصحية التي تواجه العاملين في صناعة الملابس الجاهزة ؟

• ما طرق مواجهة هذه المشكلات بناء علي توجهات الدراسة ؟

\section{أهمه التواسمة}

• الأهمية العلمية: إبراز مجال العلوم الإنسانية بدراسة عن الآثار النفسية والصحية لتلوث الهواء بصناعة الملابس الجاهزة .

• الأهمية التطبيقية: إمكانية التحكم في مصادر تلوث الهواء الناتج من صناعة الملابس الجاهزة ووضع الأساليب الوقائية والعلاجية لمواجهة هذه الظاهرة للحد من التلوث البيئي والمحافظة على صحة العاملين في هذه الصناعة و يترتب على ذلك زيادة الإنتاج.

\section{أهسا هـث التواسمة}

$$
\text { تثير أهداف الدراسة إلى: }
$$

• التعرف علي المشكلات النفسية التي تواجه العاملين في صناعة الملابس الجاهزة

• التعرف على المشكلات الصحية التي تواجه العاملين في صناعة الملابس الجاهزة .

• التعرف علي طرق مواجهة هذه المشكلات بناء" علي توصيات الدراسة. 


\section{مشور القراسلا}

تتمثل حدودالدراسة في الآتي:

المجال المكاني: مصنع اسبرانزا لصناعة الملابس الجاهزة، المدينة الصناعية الأولي، مدينة

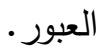

المجال البشري: عينة مكون من (ro ( ) عامل من الأقسام المختلفة داخل المصنع ( تجهيز -

تشطيب- صباغة- نسيج- إدارة).

المجال الزمني: بدأ الباحثون في جمع الاسس النظرية للاراسة والاطلاع علي الدراسات السابقة، ودارسة العناصر التي لم تتتاولها تللك الدراسات ثم العمل علي الجانب التطبيقي في

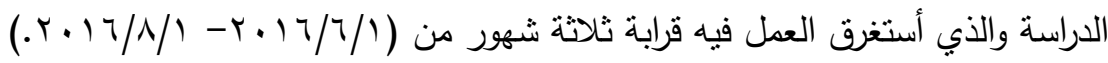

\section{هزوضر الصواسما}

• توجد علاقة ذات دلالة معنوية بين العاملين بقسم النسيج وقسم الصباغة في متوسطات درجاتهم علي مقياس إيزنك للشخصية. • توجد علاقة ذات دلالة معنوية بين العاملين بقسم النسيج وقسم والتجهيز في منوسطات درجاتهم علي مقياس إيزنك للشخصية. • توجد علاقة ذات دلالة معنوية بين العاملين بقسم النسيج وقسم والتنطيب في متوسطات درجاتهم علي مقياس إيزنك للثخصية.

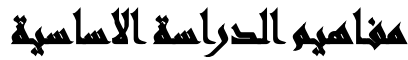

الملابس الجاهزة: ملابس الجاهزة أو ملابس جاهزة للارتداء هي كلمة يقصد بها الملابس المصنعة والجاهزة للبيع كمنتج نهائي في أحجام موحدة. وكانت بذور ما يطلق عليه الآن

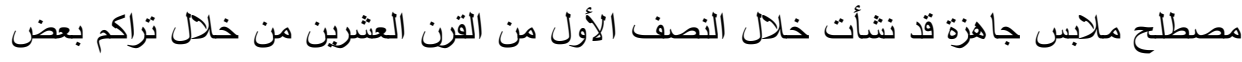

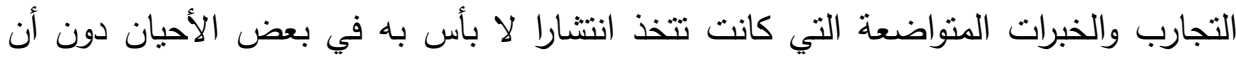
تكون بنفس الإنتاج الضخم الذي ستعرفه في النصف الثاني من هذا القرن.

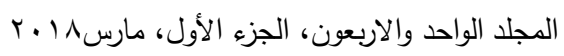


فبسبب التقدم التكنولوجي كانت الولايات المتحدة الأمريكية السابقة في نهج هذا الأسلوب الصناعي من خلال نوحيد أحجام الزى العسكري للجيش الأمريكي خلال الحرب العالمية العية الأولى.

( http://ar.wikipedia.org/w/index.php?title) مفهوم الآثار النفسية: عرفها كل من: بيرت (9VV ( )): هي نتائج تتمخض عن ظاهرة اجتماعية أو نفسية تترك صداها على الحالة النفسية للفرد لوجود العلاقة المتفاعلة بين الظاهرة الاجتماعية والظاهرة النفسية.

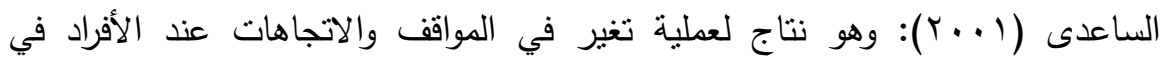
القضايا والقيم وأنماط السلوك من خلال المعلومات الصحيحة أو المشوهة أو حتى الكاذبة للتغير

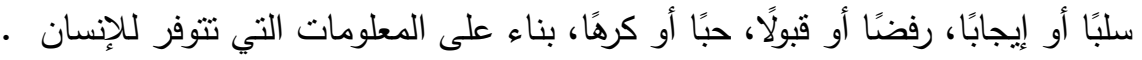
المشكلات الصحية: المشكلات الصحية في الاصطلاح: يعرفها إبراهيم شوقي عبد الحميد في بحثه مشكلات طلبة جامعة الإمارات العربية المتحدة، بأنها الاضطرابات الصحية أو الحسية

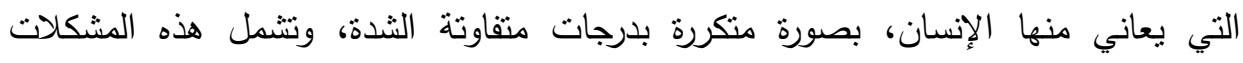

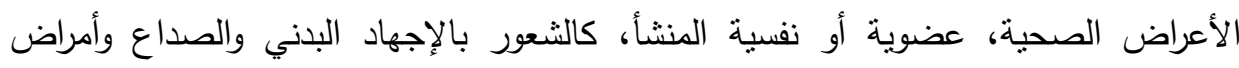
الضغط والقلب، كما تشمل ضعف الحواس وغيرها، مما يهدد التوازن النفسي والعضوي للإنسان ويحول دون توافقه مع ذاته وبيئته الفيزيقية والاجنماعية. والحالة الصحية الجيدة هي حالة اكتمال السلامة البدنية والعقلية والنفسية وليس مجرد الخلو من الأمراض أو الوهن في حال عدم تحقق تلك الشروط، يمكن اعتبار المرء يعاني من هن مشكلات صحية.

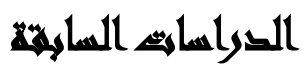

دراسة أماني أحمد محمد(9999 ( 9 ) ): بعنونان: أثر التلوث البيئي علي النشاط الاجتماعي والاقتصادي للقوي العاملة في بحيرة المنزلة. هدفت الدراسة إلي الكثف عن العلاقة بين تلوث بحيرة المنزلة والنشاط الاجتماعي في

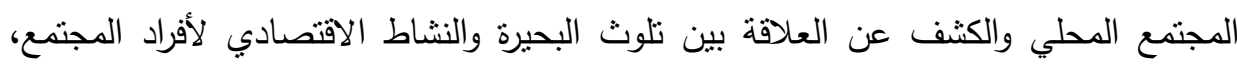

$$
\text { المجلد الواحد والاربعون، الجزء الأول، مارس^ا بـr }
$$


تتفق هذه الدراسة مع الدراسة الحالية في توضيح القصور في إدراك المجتمع إلى حد ما لآثر التلوث على صحتهم،

دراسة لي لان( • ( • ب): بعنوان (آثار درجة حرارة الهواء علي العاملين في المصانع وتقييمها بمعدل الإنتاج وعبء العمل)(أوكرانيا). هدفت الدراسة إلي التعرف علي تأثثر درجة

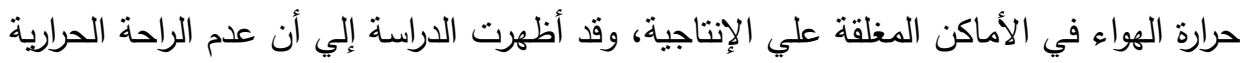
الناتجة عن ارتفاع درجة حرارة الهواء تؤثثر سلبا علي إنتاجية العاملين.

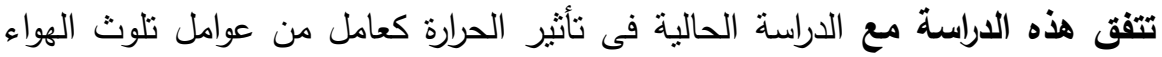
على بعض العاملين فى المجالات المختلفة.

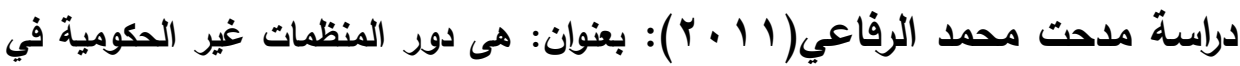
صنع السياسات العامة للبيئة مع التطبيق علي قضية تلوث الهواء في (ج. م. ع. ع.) هافت الدراسة إلي التعرف علي الدور الذي تلعبه المنظمات الحكومية وغير الحكومية حاليا في عملية صنع السياسات العامة، والوقوف علي السياسات التي تم اتخاذها لمواجهة مشكلة تلوث الهواء في مصر ، حيث اعتمدت هذه الدراسة علي تحليل وعرض للقوانين والوثائق المنظمة لعمل المنظمات الحكومية وغير الحكومية من جهود في سبيل مواجهة مشكلة تلوث

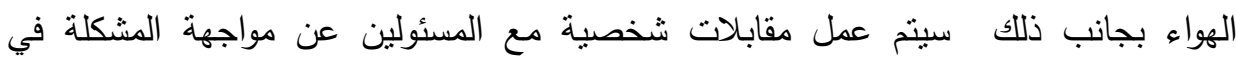
القاطعين الحكومي وغير الحكومي. تتقق هذه الدراسة مع الدراسة الحالية فى اهتمامها بموجهة تلوث الهواء وعرض القوانين اللازمة لذلك. 


\section{الإطار اللنظاري للتراسة}

التلوث الهوائي: مقدمة: يعد التلوث من المشاكل الكبيرة التي يواجهها الإنسان المعاصر . لا بل وأخطرها. وهي بحاجة إلى تظافر الجهود كافة لمعالجتها والحد منها. ومما يزيد المشكلة تعقيداً إن للإنسان نفسه الدور الواضح في زيادة خطورتها من خلال نشاطاته المختلفة التي

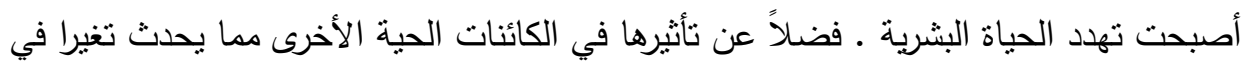
التوازن الطبيعي للبيئة ومكوناتها المختلفة الحية منها وغير الحية (مولود بهرام خضر فئر

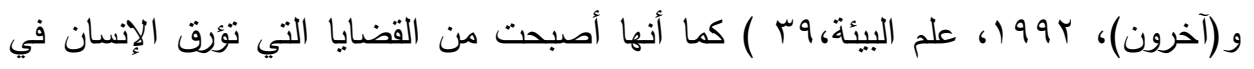
جميع المجتمعات لاسيما وأن الهواء يعتبر ضرورياً للإنسان شأنه شأن الماء، بل أكثر أهمية ألهاء

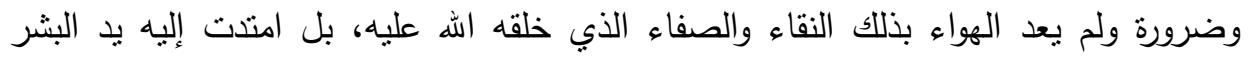
عابثة، فاختل التوازن واهتز هذا النظام الدقيق، وتلوث فكانت المعامل والمصانع ووسائل النقل ومحطات نوليد الطاقة والاستخدامات المنزلية، هذه المصادر تبث يومياً ملايين الأطنان من فن الملونات المختلفة إلي الهواء. عملية تكون التلوث: إن التلوث هو الناتج النهائي لعملية تتكون منها الفضلات أصلا وهذه العملية ذات إجراءات مختلفة وذللك لاختلاف أنواع الفضلات أو اختلاف أنواع التلوثو هناك عدة طرق للسيطرة على التلوث وفي مراحل عديدة من عملية التلوث وستؤثز طرق السيطرة هذه مباشرة في جزء من العملية وربما بشكل غير مباشر في الأجزاء الأخرى.

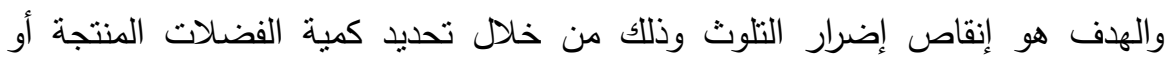
معاملة الفضلات بشكل أكثر فاعلية أو من خلال السبطرة على أسلوب وموقع دفن الفضلات أو أو

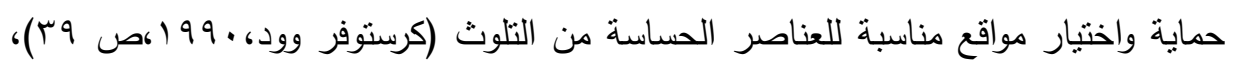

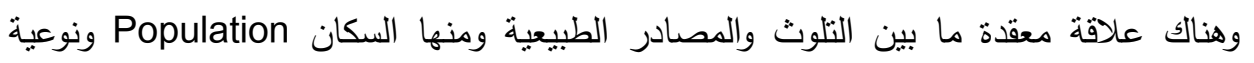

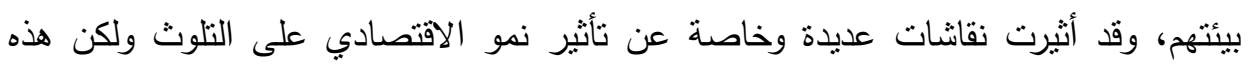

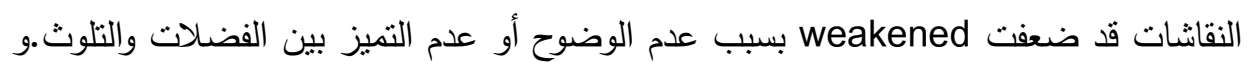

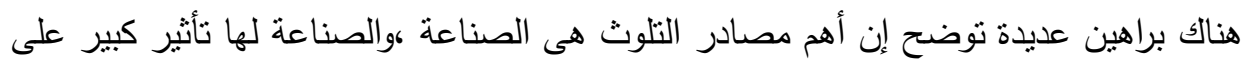


التلوث والمصانع الحديثة نسبب تلوثاً اقل من المصانع القديمة ذات نفس الإنتاج بالنسبة إلى

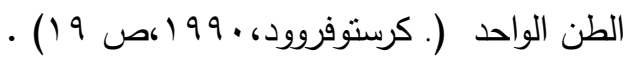

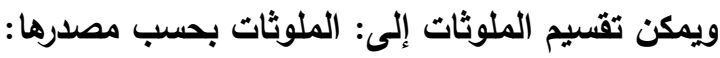

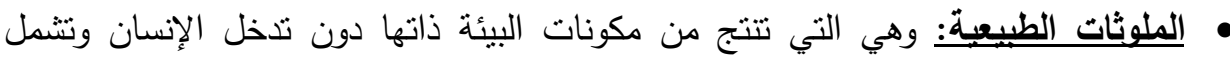
الغازات الناتجة من البراكين كثاني أوكسيد الكبريت، الأملاح في المباه، دقائق الغبار في داني

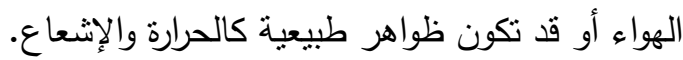
• الملوثات التكنولوجية والصناعية: وهي التي تتكون نتيجة لما استحدثه الإنسان في البيئة من تقنيات وما ابتكره من اكتشافات كذلك الناتجة عن الصناعات المختلفة والتقجيرات النووية ووسائل المواصلات.

ملوثات الإنسان والحيوان: وتتمل ما يطرحه الإنسان من فضلات نتيجة نشاطاته اليومية كالملونات الناتجة عن المدن والمجمعات السكنية التي تتمل مياه المجاري بما تحويه من المواد العضوية بدرجة رئيسية وكذلك تثمل الفضلات الحيوانية، والجدير بالذكر أن هذه الملونات

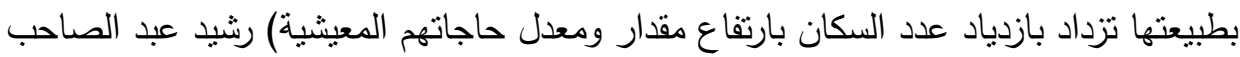
ناجي، (الأسس التخطيطية لتوقع الصناعات الملوثة وغير الملوثة في المدن العراقية)، رسالة

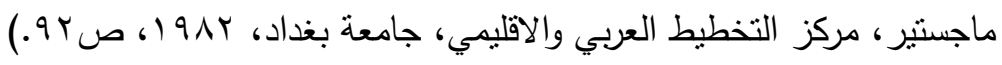

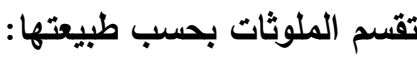
• الملوثات ذات الطيعة القفزيائية: وهي ظواهر فيزياوية مادية وتشثمل الإثعاع (وهو اثد الثدات

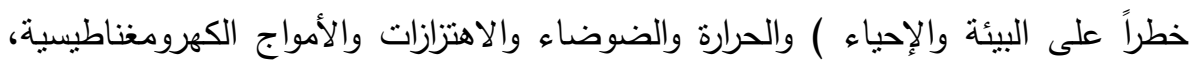

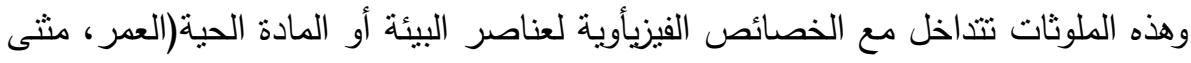
عبد الرزاق، (نظرة تحليلية للاثار البيئية للعدوان الثثلاثيني على العراق) بحث في كتاب:

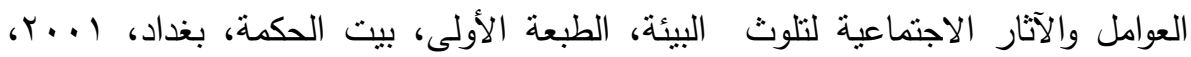

• الملوثات الطيعة الكيماوية: وهي مجموعة واسعة من الملوثات الأكثر انتشاراً في البيئة، وتشمل عدداً كبيراً من المواد الطبيعية كالنفط ومشتقاته والزيوت والثحوم والسموم الطبيعية

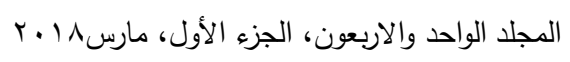


والرصاص والزئبق والغازات المتصاعدة من البراكين وعددا كبيرا من المواد المصنعة

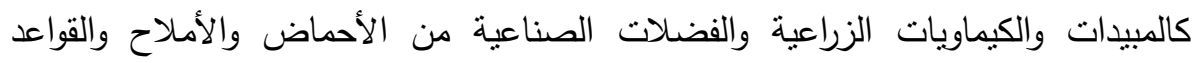

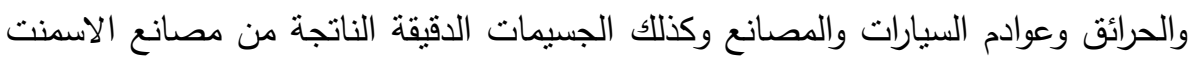
والكيماويات السائلة عندما تلقى في التربة أو الماء (.العمر، مثثى عبد الرزاق، مصدر واتئر

• الملوثات الإحيائبة: وهي كائنات حية مجهرية في الغالب وتعمل على تغير بعض الصفات

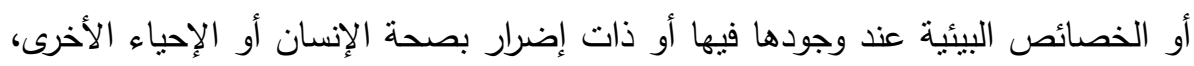
وهي على وفق الأسس العلمية لعلم البيئة فإنها تعد من المكونات الإحبائية الطبيعية، ومنها

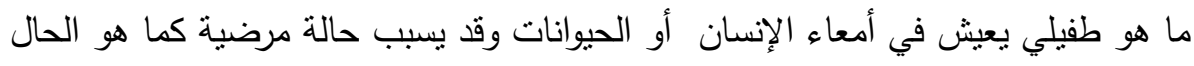
بالنسبة للطفيليات المعوية أو ليس له نأثثر صحي ضاء الإنيان كما في حالة العديد من البكتريا

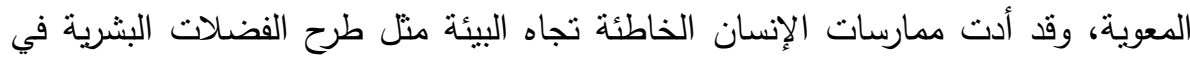

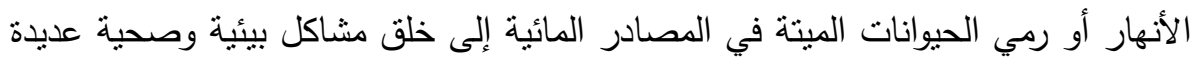
وبالتالي تحمل هذه الأحياء إلى ملوثات بيئية ولذلك فان تعبير الملوثات الإحيائية يقتصر على المسببات المرضية فقط كالبكتريا والطفيليات والفطريات والفيروسات وغيرها.

\section{إجباءاهي التراسما}

منهج الاراسة: استخدم الباحثون المنهج الوصفي المقارن، من خلال دراسة ميدانية ونظرية تتمثل في تحديد المقاييس والبحوث والدراسات وأيضا الأبحاث المتعلقة بالآثار الصحية والنفسية

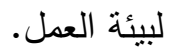
أدوات الدراسةة: - n

الاستبيان الصحي: إعداد الباحثون: قام الباحثون بإعداد قائمة بأهم المشكلات الصحية

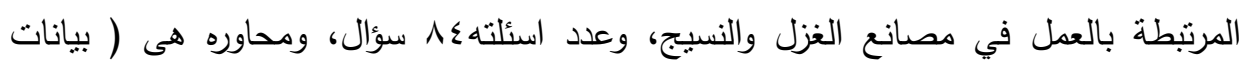
شخصية - بيانات خاصة بالسكن - بيانات مرتبطه بالعمل - بيانات مرتبطة بالسلامة المهنية - الرعاية الصحية - بيانات مرتبطة بمشكلات الضوضاء - بيانات مرتبطة بمشكلات الحرارة - 
بيانات مرنبطة بمشكلات الغبار - بيانات مرتبطة بمشكلات الإضاءه - تحليل اسباب الحوادث

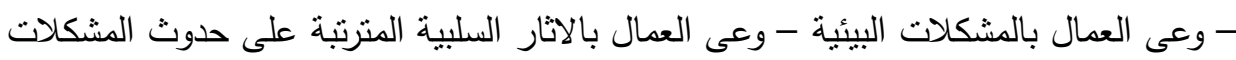

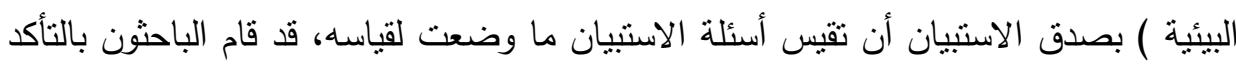
من صدق الاستبيان الصحي وذلك من خلال عرضه على مجموعة من الخبراء و قاموا بتحكيم

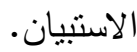

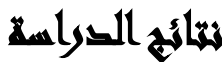

الفرض الأول: نوجد علاقة ذات دلالة معنوية بين قسم النسيج وقسم الصباغة في متوسطات درجاتهم علي مقياس إيزنك للشخصية. عدم وجود فروق بين قسم النسيج وقسم الصباغة في بعد الذهانية حيث كان منوسط قسم

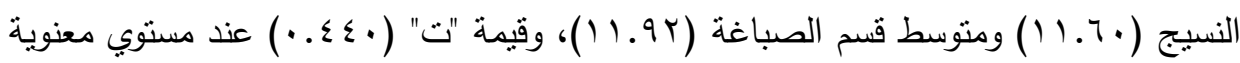
أكبر من (0. . •)، وهي بذلك تكون غير دالة إحصائياً.

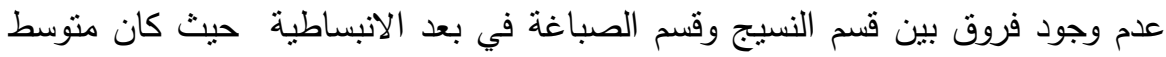

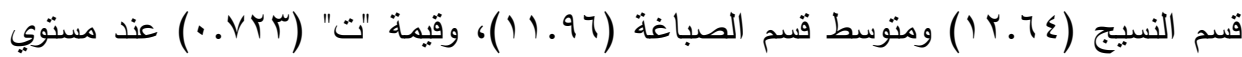
معنوية أكبر من (0. . .)، وهي بذللك تكون غير دالة إحصائياً.

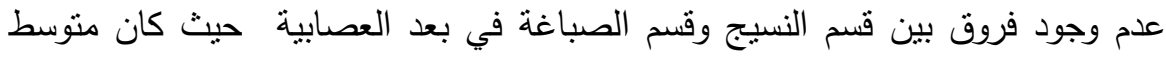

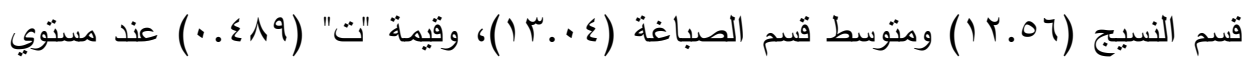
معنوية أكبر من (0. . .)، وهي بذلك تكون غير دالة إحصائياً. عدم وجود فروق بين قسم النسيج وقسم الصباغة في بعد الكذب الب حيث كان منوسط قسم

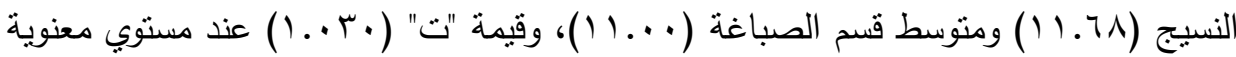
أكبر من (0. . •)، وهي بذلك تكون غير دالة إحصائياً. عدم وجود فروق بين قسم النسيج وقسم الصباغة في مقياس إيزنك حيث كان منوسط قسم

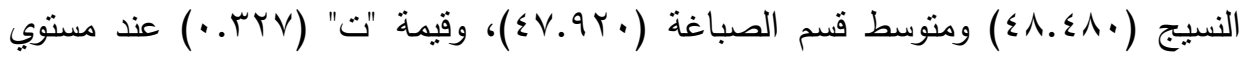

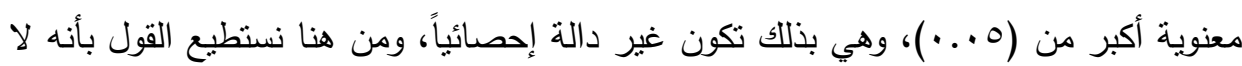
توجد فروق بين قسم النسيج وقسم الصباغة في مقياس ايزنك.

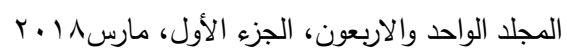


جدول( ) : اختبار T-Test يوضح الفروق بين قسم النسيج وقسم الصباغة

\begin{tabular}{|c|c|c|c|c|c|c|c|}
\hline \multirow{2}{*}{ المعنويةية } & \multirow{2}{*}{ الدستويةً } & \multirow{2}{*}{ "تيمة } & \multicolumn{2}{|c|}{ الصباغة } & \multicolumn{2}{|c|}{ قُم النسيج } & \multirow{2}{*}{ مقياس } \\
\hline & & & الانحراف & المتوسط & الانحراف & المتوسط & \\
\hline غَغِّ & .077 & $\cdot . \leq \varepsilon$. & Y.AVI & $11.9 r$ & T.YTY & 11.7. & الذهانية \\
\hline غالَّة &.$\Sigma \Sigma V T$ &.$V Y T$ & 7.117 & 11.97 & r.OTr & $1 Y .7 \varepsilon$ & الاتبساطية \\
\hline دالِّة & $.7 T V$ & $\cdot . \leqslant \wedge 9$ & $r . . r$. & $1 T . . \varepsilon$ & r.Атr & $1 Y .07$ & العصابية \\
\hline غالثية & . & $1 . . r$. & r.IV9 & $11 \ldots$ & Y. $\Sigma \vee q$ & 11.71 & الكذب \\
\hline دالثير & $\therefore v \leqslant 0$ & . TYV & $7.0 Y 01$ & $\varepsilon V .9 Y$ & $0.0 \mathrm{HAV}$ & $\{\wedge . \varepsilon \wedge$ & ايزياسالي \\
\hline
\end{tabular}

إنبات صحة الفرض القائل: توجد علاقة ذات دلالة معنوية بين قسم النسيج وقسم الصباغة في متوسطات درجاتهم علي مقياس إيزنك للشخصية. الفرض الثانى: توجد علاقة ذات دلالة معنوية بين قسم النسيج وقسم والتجهيز في متوسطات درجاتهم علي مقياس إيزنك للشخصية.

عدم وجود فروق بين قسم النسيج وقسم التجهيز في بعد الذهانية حيث كان منوسط قسم إيت إنه

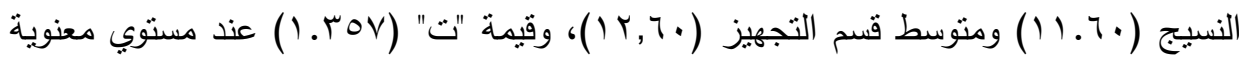
أكبر من (0...)، وهي بذلك تكون غير دالة إحصائياً، ومن هنا نستطيع القول بأنه لا توجد

$$
\text { فروق بين فسم النسيج وقسم التجهيز في بعد الذهانية. }
$$

عدم وجود فروق بين قسم النسيج وقسم التجهيز في بعد الانبساطية حيث كان منوسط قسم

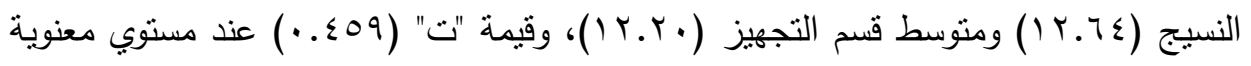

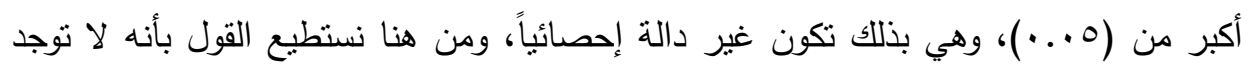

$$
\text { فروق بين قسم النسيج وقسم التجهيز في بعد الانبساطية. }
$$

وجود فروق بين قسم النسيج وقسم التجهيز في بعد العصابية حيث كان منوسط قسم

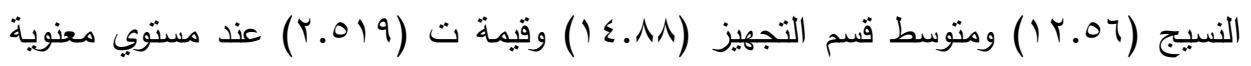
(0 . •.)، وهي بذلك تكون دالة إحصائياً، وعلى هذا الأساس توجد فروق بين قسم النسيج وقسم 
التجهيز في بُعد العصابية لصالح قسم التجهيز مما بدل علي ارتفاع في درجات العصابية وانخفاظ في درجات الانبساط مما يدل على تقلب المزاج والإنطوائية. عدم وجود فروق بين قسم النسيج وقسم التجهيز في بعد الكذب حيث كان منوسط قسم

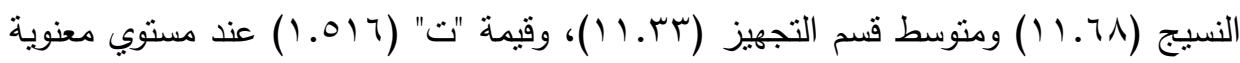
أكبر من (0...)، وهي بذلك تكون غير دالة إحصائياً، ومن هنا نستطيع القول بانه لا توجد

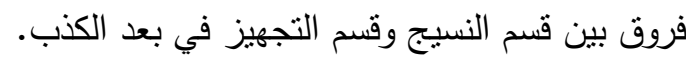

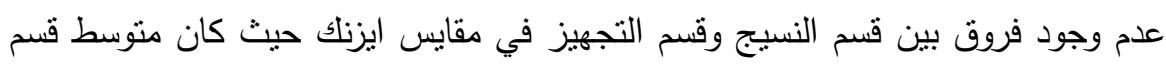

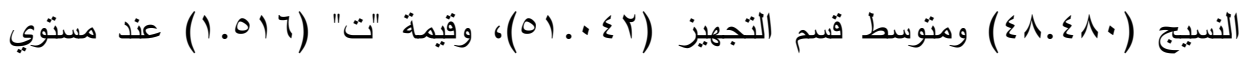
معنوية أكبر من (0. . •)، وهي بذلك تكون غير دالة إحصائياً، ومن هنا نسنطيع القول بانه لا توجد فروق بين قسم النسيج وقسم التجهيز في مقياس ايزنك. جدول(r): اختبار T-Test يوضح الفروق بين قسم النسيج وقسم التجهيز

\begin{tabular}{|c|c|c|c|c|c|c|c|}
\hline \multirow{2}{*}{ المعنتوية } & \multirow{2}{*}{ مستويلً } & \multirow{2}{*}{ "قيمة" } & \multicolumn{2}{|c|}{ التجهيز } & \multicolumn{2}{|c|}{ قُم النسيج } & \multirow{2}{*}{ مقياس } \\
\hline & & & الانحراف & المتوسط & الانحراف & المتوسط & \\
\hline غير &. .111 & $1 . \mathrm{rov}$ & r.qT. & M.T. & T.YTT & 11.7 & الذهانية \\
\hline غالة & $.7 \leqslant \wedge$ & $\therefore \leqslant 09$ & r.ror & IY.r. & T.OTY & IT.TE & الانبساطية \\
\hline. .00 &. .10 & $r .019$ & Y.0.0 & $1 \varepsilon . \wedge \wedge$ & r.Атr & $1 Y .07$ & العصابية \\
\hline غير & שTד. & $\because \leqslant \Lambda 1$ & r.070 & س. 11. & r. $\leqslant \vee q$ & 11.71 & الكذب \\
\hline غالنة & . & 1.017 & $7 . Y \vee 97$ & $01 . . . \leqslant Y$ & $0.0 \mathrm{HNV}$ & $\varepsilon \wedge . \leqslant \wedge$. & ايزّنك \\
\hline
\end{tabular}

إثبات صحة الفرض القائل: نوجد علاقة ذات دلالة معنوية بين قسم النسيج وقسم التجهيز في متوسطات درجاتهم علي مقياس إيزنك للشخصية الفرض الثالث: توجد علاقة ذات دلالة معنوية بين قسم النسيج وقسم والتتطيب في متوسطات درجاتهم علي مقياس إيزنك للشخصية. عدم وجود فروق بين قسم النسيج وقسم التشطيب في بعد الذهانية حيث كان منوسط قسم

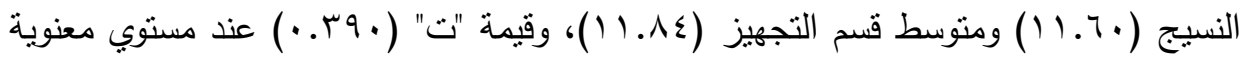

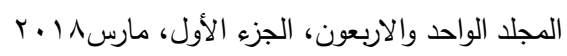


أكبر من (0...)، وهي بذلك تكون غير دالة إحصائياً، ومن هنا نستطيع القول بانه لا توجد فروق بين قسم النسيج وقسم التشطيب في بعد الذهانية.

عدم وجود فروق بين قسم النسيج وقسم النتطيب في بعد الانبساطية حيث كان منوسط

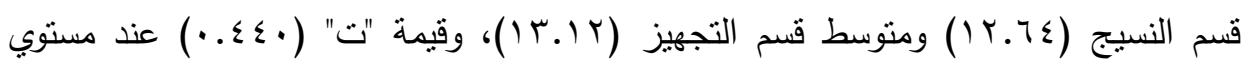
معنوية أكبر من (0...)، وهي بذللك تكون غير دالة إحصائياً، ومن هنا نستطيع القول بانه لا لا نوجد فروف بين قسم النسيج وقسم التشطيب في بعد الانبساطية. عدم وجود فروق بين قسم النسيج وقسم التشطيب في بعد العصابية حيث كان منوسط قسم

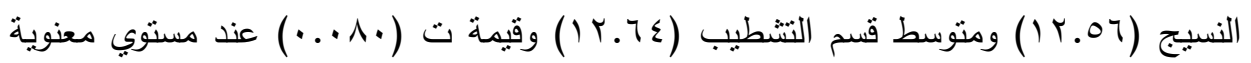
أكبر من (0. . •)، وهي بذلك تكون غير دالة إحصائياً، ومن هنا نستطيع القول بانه لا توجد فروق بين قسم النسيج وقسم التشطيب في بعد العصابية. وجود فروف بين قسم النسيج وقسم التشطيب في بعد الكذب حيث كان منوسط قسم النسيج

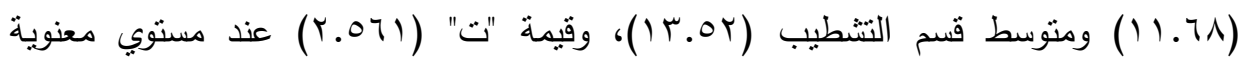
(0...)، وهي بذلك تكون دالة إحصائياً، وعلى هذا الأساس توجد فروق بين قسم النسيج وقسم

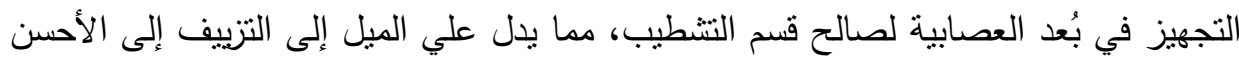

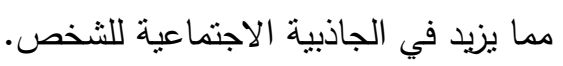
عدم وجود فروق بين قسم النسيج وقسم النتطيب في مقاييس ايزنك حيث كان متوسط قسم

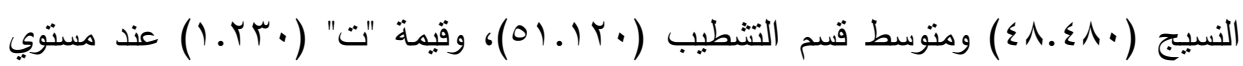
معنوية أكبر من (0. . •)، وهي بذلك تكون غير دالة إحصائياً، ومن هنا نستطيع القول بانه لا لا توجد فروق بين قسم النسيج وقسم النتطيب في مقياس ايزنك. 
جدول(r): اختبار T-Test يوضح الفروق بين قسم النسيج وقسم التشطيب

\begin{tabular}{|c|c|c|c|c|c|c|c|}
\hline \multirow{2}{*}{ المعنتويةي } & \multirow{2}{*}{ مستويةً } & \multirow{2}{*}{ "قيمة } & \multicolumn{2}{|c|}{ التشطيب } & \multicolumn{2}{|c|}{ قسم النسيج } & \multirow{2}{*}{ مقياس } \\
\hline & & & الانحراف & المتوسط & الانحراف & المتوسط & \\
\hline غيرِ &. .791 & .rq. & T.110 & $11 . \wedge \varepsilon$ & T.YTT & 11.7. & الذهانية \\
\hline غيرة & 政 &..$\leqslant \leqslant$. & $\varepsilon .17 V$ & $1 T .1 Y$ & T.OTr & IT.TE & الانبساطية \\
\hline دالثِّ & $.94 \mathrm{~V}$ & $\ldots \wedge$. & r.r.. & IT.TE & T.人тT & $1 T .07$ & العص \\
\hline. .00 & $\ldots 1 \leq$ & Y.071 & Y.7.. & Tr.or & $\varepsilon \vee q$ & 11.71 & الكذب \\
\hline غير & . YYO & 1.rT. & Q.АTVq & $01.1 \%$. & $\Lambda \mathrm{V}$ & $\leqslant \wedge$ & يقياس اجمالى ايزنك \\
\hline
\end{tabular}

إثبات صحة الفرض القائل: توجد علاقة ذات دلالة معنوية بين قسم النسيج وقسم والتتطيب في

متوسطات درجاتهم علي مقياس إيزنك للشخصية.

\section{تموسياته الدوالمة}

• تفعيل دور الأمن الصناعى والسلامة والصحة المهنية فى حماية العمال من المخاطر العمل

المحيطة بهم.

• ضرورة اهتمام المسئولين وأصحاب المؤسسات الصناعية بتزويد العمال بأدوات الوقاية من

التلوث ( الكمامات - سداد الأذن - النظارات المهنية - القفازات وبدل العمل وغيرها )

لحماية من مخاطر التلوث البيئى لبيئة العمل.

التدريب على استعمال أدوات الوقاية من التلوث وتفعيل سياسة الثواب والعقاب فى أستعمال

الادوات التى تقى العمال من النلوث.

• ضرورة نوافر أجهزة اكثر فى المصنع (فلاتر - مرشحات - شفطات - تكيفات ) لتقليل

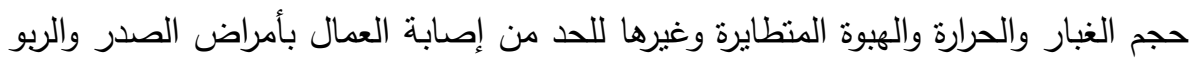

والحساسية وضيق التتفس.

التاكيد على توقيع الكثف الطبى الإبتدائى والكثف الطبى الدورى للعمال لإكتثاف ظهور

أى مرض مهنى منذ بدايته واتخاذ الإجراءات اللازمة لمعالجته.

المجلد الواحد والاربعون، الجزء الأول، مارس^ بـr 


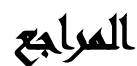

مجدي أحمد محمد عبد الله(؟ ..ب): علم النفس الصناعي بين النظرية والنطبيق، دن، دار المعرفة الجامعية، الإسكندرية.

كرستوفر وود( ع^91): تخطيط المدن والسيطرة على التلوث، ترجمة مضر خليل العمر، مطبعة جامعة البصرة، بغداد.

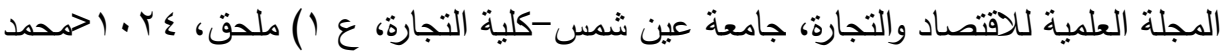
الحناوي آخرون، أساسيات السلوك التنظيمي، الاسكندرية، الدار الجامعية،

محمد القريوني( . . ب): السلوك التنظمي" دراسة للسلوك الأنساني الفردي والجماعي في

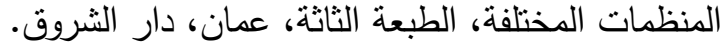

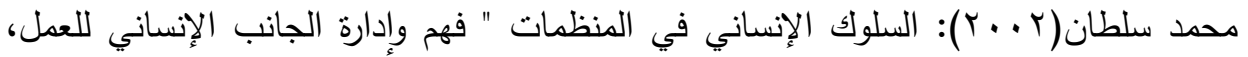

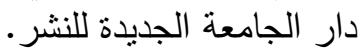

محمد عبد الجواد( . . † ) ) أسرار النجاح ومنطلقات التميز، ،الطبعة الأولى، مصر، دار البشير

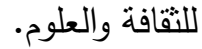

Anita Underwood "Coping with on-the-job stress" Black Entrprise (Electronic Version) ‘August. 1992

Athkinson ‘C.Atkinson ‘ \& R.Hilgard ‘Introduction to Psychology ‘5th ed 
أحمد مصطفى العتيق وآخرون

\title{
THE PSYCHOLOGICAL AND HEALTH IMPACTS OF AIR POLLUTION OF READY-ON CLOTHING INDUSTRY
}

\author{
Al-Atiq, A. M. ${ }^{(1)}$; Shoman, A. E. E. ${ }^{(2)}$ and Mohamed, S, M. \\ 1) Institute of Environmental Studies \& Research, Ain Shams University \\ 2) Faculty of Medicine, Ain Shams University
}

\begin{abstract}
After the industrial revolution the interest of the countries especially the developed ones increased with the environmental pollutants that affect the health of the workers.

Environmental pollutants were classified in the work environment as the cause of their pollutants.

These pollutants are called the physical pollutants in the work environment.

These factors affect the health of the individual who is exposed as a result of their properties And not as a result of the chemical reaction between these factors and the body tissues where workers are exposed at the workplace

to many of these risks and pollutants 'which lead to the incidence of occupational diseases and accidents in work accidents and injuries and the most important physical hazards (natural) Noise rheat clighting and ventilation.

The study aims to identify some of the health and psychological problems associated with air pollution in spinning and weaving factories.

The researcher chose the sample of the study from one of the spinning and weaving factories (Esperanza Factory for Ready Made Garments).

The total number of workers was (125) their (25) Each of the five departments (finishing - processing - management - dyeing - weaving) ‘ where the researcher designed a questionnaire on the health problems
\end{abstract}

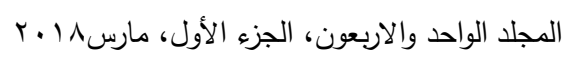




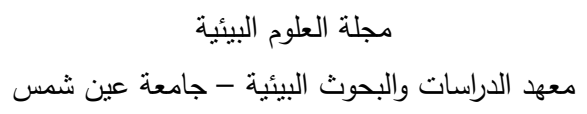

associated with work in textile factories 'the questionnaire of environmental risk perception questionnaire 'the professional pressure scale 'EISANQ Personal scale sto collect the data And application of the Sample Study .

This study belongs to a number of descriptive studies that rely on descriptive methods.

The study deals with some theories of environmental psychology that deal with the influence of psychological and physical factors in the work environment 'such as the theory of environmental hardship 'the curve of arousal sthe environmental hardship.

The researcher treated the data obtained statistically using T-Test ، Analysis of variance (ANOVA).

The study concluded to several conclusions the most important:

That there are no differences between the averages of the four sections on the occupational stress scale.

There are differences between the averages of the four sections on the environmental risk questionnaire.

There are differences between the averages of the four sections of the health questionnaire.

There is a relationship of significant relativity between the finishing department and the management department in their average scores on the EISANQ scale. 